\title{
A randomised control trial to evaluate messages that promote limit setting and the impact of limits on online gambling behaviour (pre-print)
}

\author{
Robert M. Heirene ${ }^{1} \&$ Sally M. Gainsbury ${ }^{1}$ \\ ${ }^{1}$ Brain \& Mind Centre, School of Psychology, Science Faculty, University of Sydney, Australia
}

\begin{abstract}
Background \& Aim: Deposit limits may be an effective strategy for minimising the harm associated with online gambling. However, voluntary uptake of these tools is low. This study aimed to compare the effectiveness of different messages to increase use of deposit limits by customers of online gambling sites and to examine the effects of limit setting on gambling behaviour. Design: A pre-registered, naturalistic randomised control trial was conducted wherein customers were sent varied messages by online gambling operators. Setting: Four online Australian sports and racing wagering websites. Participants: 31,989 wagering customers (reduced to 26,516 after screening for eligibility) who had placed bets on at least five days during the last 30. Interventions: Messages were sent via email or in-account notification and were designed to either: [1] be informative, describing the availability and purpose of the tool, [2] highlight the benefits other people receive from using the tool (social messages), or [3] promote the benefit individuals could receive from using the tool (personal messages). Comparators: A control group of customers who did not receive messages was monitored for comparison. Measurements: We collected customer account data for 90-days pre- and postmessages. Findings: $161(0.71 \%)$ customers set a deposit limit within five days of messages being sent. Those sent messages via in-account notification were more likely to set limits than those sent messages via email. Differences in message content had little effect on deposit limit uptake. Customers who set limits showed significantly greater decreases in average daily wager amount, the SD of average daily wager, net loss, and betting intensity compared to a randomly selected subsample of non-limit-setters. Conclusions: Customer messages are inexpensive and can lead to small but impactful increases in the uptake of deposit limits on gambling sites. Setting deposit limits was associated with decreased gambling intensity, expenditure, and losses and may therefore be an effective harm-reduction strategy.
\end{abstract}

Please note that this a pre-print, or an article published online pre-peer review.

Keywords: pre-commitment; online gambling; responsible gambling; consumer protection tools; sports betting.

\section{Introduction}

Individuals who use online gambling websites now have access to various consumer protection tools (CPTs) or "responsible gambling tools" to help them manage their spending, including the ability to set limits on how much they can deposit into their account ("deposit limits" or precommitment), access to "activity statements" outlining gambling expenditure, and the ability to temporarily block access to their accounts using "timeouts" [ 24 hours to $\geq 6$ months; $(1,2)$. Surveys of players who have used CPTs in Australia (1), Sweden (2), Finland (3), and Norway (4) have found the tools are mostly positively perceived. However, rigorous empirical evaluations of how CPT use effects actual gambling behaviour, particularly over time, are lacking. A few studies have examined the impact of setting deposit limits, which have been associated with reductions in the number of betting days, total number of bets, the overall amount wagered (5) and, in the most "intense" players, reduced gambling intensity (6) and expenditure (7). In contrast, studies have found no effect of limit setting on average bet size (5) or on net loss (8). However, these studies have predominately focused on single European operators (6-8) or are now outdated in the current online context (5), limiting the extent to which their findings can be extrapolated to customers across international online gambling sites.

Despite their positive perception and harm-reducing potential, voluntary engagement with CPTs is low. In Australia, only $24.5 \%$ of 564 online wagering customers surveyed by Gainsbury et al. in 2019 (1) had ever used deposit limits and $8.1 \%$ had used time-outs. The UK Gambling Commission found only $9 \%$ of 6,425 gamblers surveyed reported using limit setting and 3\% used timeouts in 2018 (9). Understand- 
ing why consumers use CPTs may help to increase uptake. Procter et al. (10) explored whether the Theory of Planned Behaviour (TPB) $(11,12)$ could explain CPT use among Australian online wagerers. Consistent with the TPB, the authors found positive attitudes and subjective norms, along with previous CPT use, were positively correlated with the intention to use CPTs, which subsequently predicted actual tool use. However, the third TPB construct, perceived behavioural control, was not predictive of intentions to use CPTs. A subsequent study confirmed the role of attitudes and perceived behavioural control but not subjective norms in predicting intention to use strategies to self-manage gambling, with intention predicting behaviour (13).

The UK Government's Behavioural Insights Team (14) attempted to overcome low rates of volitional CPT use on UK gambling sites by using customer messages to encourage CPT uptake. In two trials with different online gambling sites, they found differences in message content (e.g., self-reflection content vs. normative feedback) had little impact when encouraging CPT use. The only factor associated with significant increases in CPT use was reduced friction (i.e., fewer number of clicks/steps required to access the tools from the message).

Using the knowledge gained from preliminary research on CPT use, the aim of the present study was to explore the value of different message types for increasing deposit limit uptake among customers from four wagering sites in Australia and to investigate the impact of limit setting on gambling behaviour. We focused on promoting deposit limits given their relatively low rates of use $(1,9)$ and potential relevance to a wide customer base when compared with more restrictive tools (e.g., timeouts, self-exclusion). Based on Procter and colleagues' (10) finding that CPT use was motivated by positive attitudes towards the tools and subjective norms (i.e., the sense that others use and think favourably of

Trial registration: The trial preregistration, full study protocol, pilot study reports, materials, data analysis code, and all analysis outputs are all shared on this project's Open Science Framework page: https://osf.io/6dpkw/.

The authors made the following contributions. Robert M. Heirene: Conceptualization, Data curation, Formal analysis, Methodology, Software, Visualization, Writing - Original Draft, Writing - Review \& Editing, Project administration; Sally M. Gainsbury: Conceptualization, Funding acquisition, Methodology, Supervision, Writing - Review \& Editing.

Correspondence concerning this article should be addressed to Robert M. Heirene, Gambling Treatment \& Research Clinic, Brain $\&$ Mind Centre, University of Sydney, 94 Mallett St, Camperdown, Sydney, NSW, Australia, 2050. E-mail: robert.heirene@ sydney. edu.au them), we compared the effectiveness of messages that [1] highlighted the benefits to the individual of setting a deposit limit (e.g., managing spending [labelled "positive personal perception message"]) and [2] highlighted the positive social perception of the tool (i.e., others use and like deposit limits ["positive social perception message"]), as well as [3] a message including a description of deposit limits ("informative message") for comparison with the theoretically informed messages. In our study preregistration we outlined three hypotheses:

- $\left[\mathrm{H}^{1}\right]$ Online wagerers in the messaging conditions will be more likely than controls (i.e., those who do not receive a message) to set a deposit limit within five days of messaging (the same time period used by BIT, 2018).

- $\left[\mathrm{H}^{2}\right]$ Online wagerers who receive positive social perception messages will be most likely to set a deposit limit within five days of messaging ${ }^{1}$, followed by those who receive positive personal perception messages, and then those who receive informative messages.

- $\left[\mathrm{H}^{3}\right]$ Messages delivered via in-account notification will be more effective at increasing deposit limit uptake than those delivered via email (due to reduced friction).

We made tentative predictions regarding the effects of deposit limits on wagering behaviour based on the existing literature but did not preregister any fixed hypotheses. Our predictions were that "wagerers who set deposit limits will reduce the amount wagered and the duration ${ }^{2}$ and frequency of wagering subsequent to limit-setting (compared with baseline data), but no effect on net loss will be observed".

\section{Methods}

\section{Registration \& transparency}

A detailed study protocol was preregistered on Open Science Framework (OSF) prior to commencing the trial: https: //osf.io/6dpkw/. Unless otherwise stated, we followed the plan described in our preregistration. All deviations are reported in a transparent changes document shared on OSF and deviations affecting confirmatory analyses are reported here.

\footnotetext{
${ }^{1}$ Socially referenced messages were more positively rated overall than positive personal perception messages by gambling customers involved in pilot testing the messages. Thus, $\mathrm{H}^{2}$ is based on a combination of preliminary theory application (TRA) with descriptive work (10) and pilot testing-all strategies recommended for hypothesis development (15).

${ }^{2}$ As stated in our transparent changes document (https://osf.io/ $6 \mathrm{dpkw} /$ ), operators were unable to provide data relating to time spent gambling and therefore we were unable to investigate the effect of limit setting on this variable.
} 


\section{Design}

A pragmatic or naturalistic randomised control trial was used (16) wherein customers were unaware that the messages they received were part of a trial. This allowed us to evaluate the effectiveness of the messages for encouraging limit use in a setting with high ecological validity (17). Customers from four Australian online sports and racing wagering sites were randomly allocated by their respective operator to one of seven possible conditions, including a control group of customers who did not receive messages for comparison. A factorial approach was used whereby the intervention groups were divided according two variables resulting in six conditions: delivery method (two levels: email and in-account delivery) and message content (three levels: informative, personal, and social). The primary outcome was whether customers set a deposit limit within five days of receiving messages. A secondary outcome outlined in our protocol was message opening rates, although only three operators were able to record this data and two provided only summary figures (e.g., " $35 \%$ of customers in condition 1 opened message 1"). As a result, we diverged from our protocol by including all those who received messages in analyses of our hypotheses, regardless of whether they opened them.

\section{Participants}

Participants were account holders of Australian sports and race wagering websites. An a priori power analysis $(\alpha=.05$, $\beta=.05, d f=6$ ) for a Chi-squared analysis with a small effect size $(\varphi=0.1)$ indicated that the total sample size required was 2,086 . This was multiplied 20 times to account for the lowest estimated opening rate of messages (5\%) according to operators. This resulted in a required sample of 41,720 , which we rounded to 42,000 . One operator unexpectedly dropped out of the trial which reduced the actual sample size involved in the study to 31,989 . The processes of participant recruitment, allocation, and screening for eligibility criteria are presented in Figure 1 and the characteristics of the final sample used in tests of hypotheses are described in Table 1.

\section{Development \& pilot testing of customer messages}

We used a systematic approach to develop and pilot test the messages used in the trial, initially developing six social and six personal messages based on the findings of existing research on responsible gambling messaging (see protocol on OSF for a discussion of this literature). These were circulated to external researchers for evaluation and amended accordingly. Next, messages were subjected to consumer evaluations via an online market research platform operated by one of the gambling sites participating in the trial $^{3}$. Customers rated all messages along a 7-point Likert scale and were able

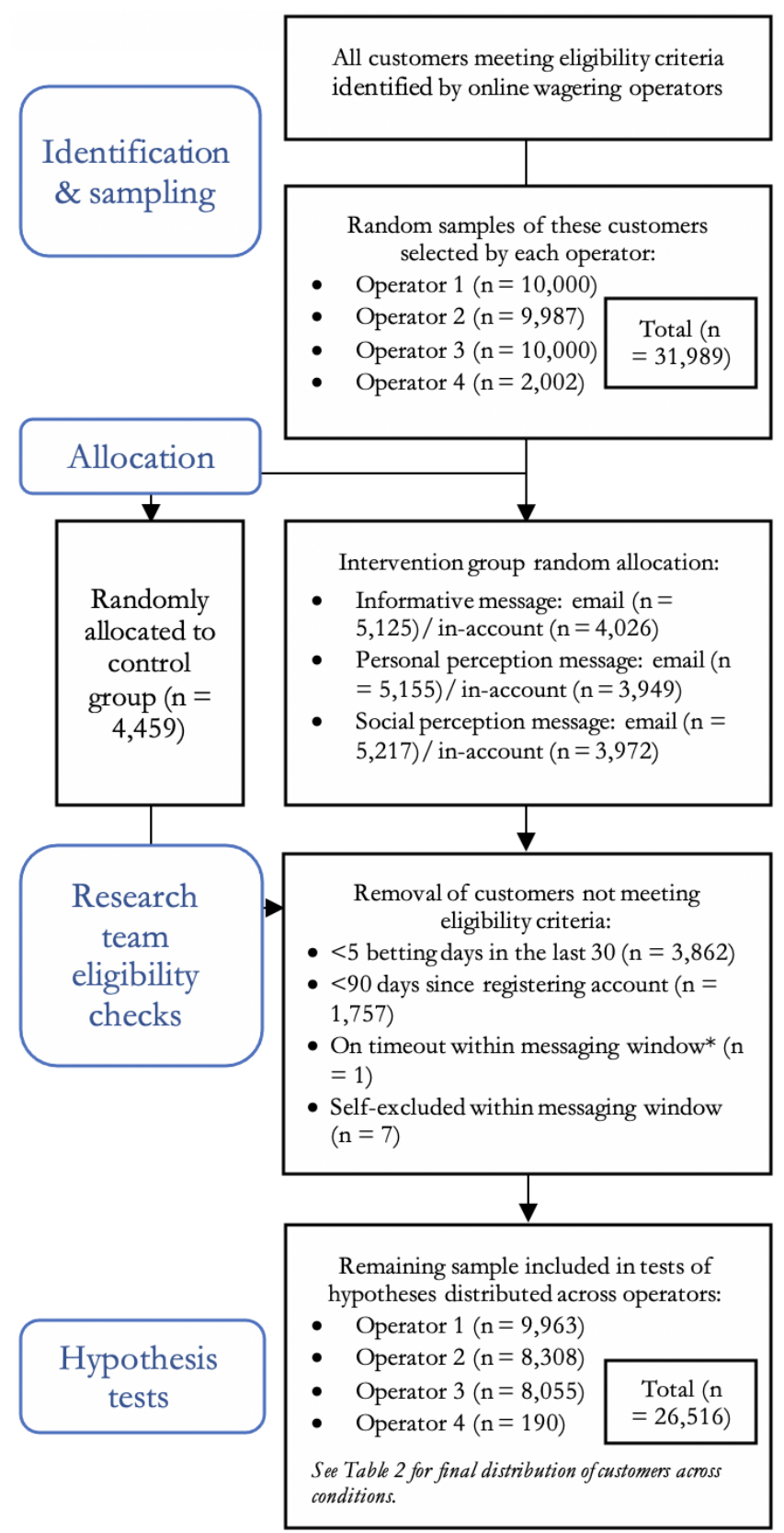

Figure 1. Flow-chart of participant recruitment \& selection. Figure legend: This CONSORT-style flowchart presents the process of identifying, selecting, and allocating customers for involvement in the trial, as well as the subsequent removal of those not meeting study eligibility criteria. Some customers may have not met multiple eligibility criteria (e.g., they held an account for $<90$ days and bet on $<5$ days). *messaging window defined as the duration between the first message being delivered and five days after the second message was delivered (i.e., 14/10/2019 - 23/10/2019).

\footnotetext{
${ }^{3}$ All those who participated in the polls used to pilot test messages were excluded from the trial.
} 
Table 1

\begin{tabular}{lclllll}
\multicolumn{7}{l}{ Demographic characteristics of participants included in tests of hypotheses } \\
\hline Condition & N & M age (SD) & Mdn age & Gender: F (\%) & Gender: M (\%) & Gender: U (\%) \\
\hline 1 & 3814 & $41.63(14.30)$ & 39.00 & $326(8.55)$ & $3041(79.73)$ & $447(11.72)$ \\
2 & 3906 & $40.42(14.14)$ & 38.00 & $311(7.96)$ & $3163(80.98)$ & $432(11.06)$ \\
3 & 3670 & $42.18(14.50)$ & 40.50 & $260(7.08)$ & $2899(78.99)$ & $511(13.92)$ \\
4 & 3981 & $40.81(13.98)$ & 38.00 & $376(9.44)$ & $3137(78.80)$ & $468(11.76)$ \\
5 & 3643 & $42.18(14.67)$ & 40.00 & $269(7.38)$ & $2839(77.93)$ & $535(14.69)$ \\
6 & 3888 & $40.67(13.86)$ & 38.00 & $320(8.23)$ & $3129(80.48)$ & $439(11.29)$ \\
7 & 3614 & $42.02(14.45)$ & 40.00 & $253(7.00)$ & $2842(78.64)$ & $519(14.36)$ \\
Total & 26516 & $41.39(14.28)$ & 39.00 & $2115(7.98)$ & $21050(79.39)$ & $3351(12.64)$ \\
\hline
\end{tabular}

Note. Conditons: 1: Control group, 2: Informative (email), 3: Informative (in-account), 4: Social (email), 5: Social (in-account), 6: Personal (email), 7: Personal (in-account); $M=$ Mean; Mdn = Median; Gender: $\mathrm{F}=$ Female, $\mathrm{M}=$ Male, $\mathrm{U}=$ Unknown.

to provide qualitative feedback on messages. Tag lines that accompanied messages and subject-lines for email messages were developed and evaluated using the same methods. Full details of this pilot work-including methods, all messages and email subject-lines tested, outcomes, and the selection process-are reported on OSF.

Based on the outcomes from polls, the message selected as the social perception message was: "Most people who use deposit limits find this helps them manage their spending" and the personal perception message selected was: "Deposit limits are a great way to manage your spending". The tag line that accompanied both of these messages was: "Keep on track with deposit limits". The email subject-lines selected were: "Keep on track" (used for the first email message sent to customers) and: "Set and forget" (used for the second message). The informative message used was: "Customers are able to set a personal limit on the amount of money deposited into their gambling account using the Deposit Limits tool, for a 24 hour, weekly, two weekly, or monthly period" (no tag line was presented with this message). This was modelled on standard operator communications regarding deposit limits.

\section{Procedure}

Operators were provided with detailed instructions on how to randomly select and allocate cohorts of customers to conditions using a random number generation procedure (18). Participants allocated to one of the intervention groups were sent the message corresponding to their condition on 14/10/2019. If they did not set a deposit limit within the ensuing five days, a second message with the same content (although with a different subject line for those sent email messages) was sent five days later on 19/10/2019. Operators were responsible for identifying customers meeting the pre-specified eligibility criteria, however, as seen in Figure 1, many customers originally identified and included in the study (particularly those by Operator 4) did not meet eligibility criteria and were removed from analyses by the research team. We requested account data for all customers from operators for a period spanning 90-days prior to the first message and 90 days following the second message (i.e., 16/07/2019-17/01/2020), including account ID, date account opened and (if relevant) closed, age, gender; postcode, any use of CPTs (including date and characteristics such as limit amount/duration), and details of transactions and wagers (e.g., date, sport, stake, payout).

\section{Ethics}

Ethical approval to undertake the trial was obtained from the University of Sydney Human Research Ethics Committee (reference number: 2018/400). Formal consent was not be obtained from customers due to the naturalistic design of the trial. All customers were users of online wagering operators and by opening accounts they provided consent to the terms and agreements of these operators, which include that they may be contacted in relation to the CPTs available to them and that their anonymised data may be shared by an authorised third party.

\section{Data analysis}

Data were provided by operators in spreadsheets and imported to the statistical programme $\mathrm{R}$ (version 4.0.2)(19) for pre-processing and analysis. We tested the study hypotheses relating to the frequency of limit setting across conditions using Chi-square analyses. To provide further information than stated in our preregistration, we supplemented each frequentist test of our hypotheses with Bayesian tests of association. 
As six confirmatory analyses were conducted, we adjusted $\alpha$ to 0.0083 using the Bonferroni correction method ${ }^{4}$.

For exploratory analyses of how deposit limits affected wagering behaviour (e.g., average daily wager), we filtered the hypothesis test sample by removing all those with $<14$ days wagering activity either side of messages due to account closures or the use of timeout and/or self-exclusion features. We also removed all non-limit-setters who set a limit in the 85 days after the post-message window of interest, leaving 153 limit setters and 22,207 non-limit-setters. We then randomly selected 153 non-limit-setters (matched for operator) for comparison. For outcomes affected by duration (i.e., net loss, betting frequency, intensity) we removed any customers with a break of $\geq 14$ days either side of messages due to account closures or CPT use. We aimed to use analyses of covariance (ANCOVAs) with change scores (i.e., the difference between the score for 90 -days pre- and post-limit) as the outcome variable and baseline scores (i.e., 90-days pre-limit) as the covariate to account for the phenomenon of regression to mean (20). Although, as data for all comparisons did not meet several statistical assumptions required for traditional ANCOVA, we undertook robust versions of the test using the "WRS2" package in R (21). Robust ANCOVAs involve the use of trimmed means and a running interval smoother (a function that attempts to approximate the true regression lines and account for their curvature) and perform well in simulated scenarios when standard ANCOVA assumptions (e.g., homogeneity of regression slopes) are not met (22). The test involves comparing the two groups' scores at multiple "design points", or around values of the covariate deemed to be "comparable" (i.e., where scores are available for $\geq 12$ participants per group $)^{5}$. To account for Type-I error risk (5 robust ANCOVAs involved 25 comparisons, 5 per test), we adjusted $\alpha$ to 0.002 (Bonferroni correction). For more details on our analysis process and the tests used, we have provided an extended data analysis section and a document presenting the annotated analysis code used with outputs on OSF: https://osf.io/6dpkw/.

\section{Results}

In total, 161 customers in the messaging groups set a deposit limit within the five days following the messages being sent $(0.71 \%$; see Figure 2 for breakdown by condition). Only three of the customers in the control group set a limit within the same time period $(0.08 \%)$. The opening rate data provided by three operators suggested emails were opened on average by $30.6 \%$ of customers (range $22.4-38.5 \%$ ). We calculated the estimated number of participants in the sample used to test hypotheses who opened emails using the condition-specific rates from the full, pre-screening samples for three the operators where data were available and using the combined mean rates from these operators for the

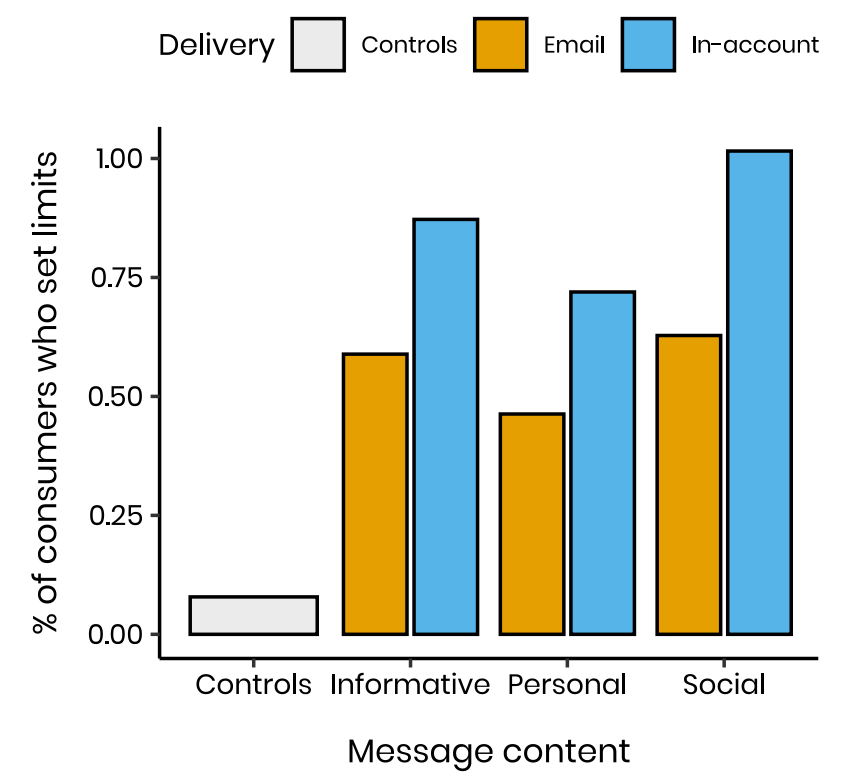

Figure 2. Rates of limit setting within each experimental condition. Figure legend: This bar chart presents the percentage of limit setters within each experimental condition. A customer was defined as a "limit setter' if they set a limit within five days of the first message or second message being sent (i.e., between 14/10/2019 \& 23/10/2019) or within the same window for controls.

fourth operator. Only including the estimated number of customers who opened emails raises the limit setting rate in the email conditions from 0.56 to $2.00 \%$, and the total rate increases from 0.08 to $1.13 \%$. Operators were unable to track in-account message opening rates, but it is likely the limit setting rate in these groups would also increase with similar adjustments.

\section{Confirmatory analyses}

Chi-square analysis found that the frequency of limit setting was significantly different between conditions, $\chi^{2}(6, \mathrm{n}=26516)=\sim 33.46, p_{<} .001$. However, the effect size was small $(\mathrm{V}=0.04,95 \%$ CIs $[0.02,0.05])$ and the Bayesian test of association produced a BF of 3e-05, supporting the null hypothesis that there was no overall association between limit setting and group. The frequency of limit setting among controls was significantly lower than

\footnotetext{
${ }^{4}$ We set $\alpha$ at 0.05 for exploratory analyses unless otherwise stated.

${ }^{5}$ In robust ANCOVA, the null hypothesis is that the trimmed mean of the outcome variable (e.g., change score for average daily wager) is equal for cohorts of the two groups who have similar covariate values (e.g., average daily wager amounts for the 90-days pre limit of $\$ 25$ or $\$ 100)$.
} 
among all message groups combined (i.e., conditions 2-7), $\chi^{2}(1, \mathrm{n}=26516)=\sim 20.11, p_{<} .001, \mathrm{OR}=9.07,[2.89,28.44]$, $\mathrm{BF}_{10}=8109.3$. Limit setting frequency was not significantly different between those in the social perception and informative message groups $\left(\chi^{2}\left(1,{ }_{\mathrm{n}}={ }_{15200)}=\sim 0.27\right.\right.$, $\left.p_{=} .601, \mathrm{OR}=1.12[0.78,1.61], \mathrm{BF}_{10}=0.006\right)$, between those in the personal perception and informative message groups $\left(\chi^{2}\left(1, n_{n}=15078\right)=\sim 0.92, \quad p=.337, \quad \mathrm{OR}=0.81[0.54\right.$, $\left.1.20], \mathrm{BF}_{10}=0.009\right)$, nor between those in the social perception message and personal perception message groups $\left(\chi^{2}\left(1,{ }_{n}=15126\right)=\sim 2.48, p_{=} .116, \mathrm{OR}=1.39[0.94,2.05], \mathrm{BF}_{10}\right.$ $=0.021)$. The frequency of limit setters was significantly greater in groups sent messages via in-account notification than in groups sent messages via email, $\chi^{2}(1, \mathrm{n}=22702)=\sim 7.25$, $p_{=} .007, \mathrm{OR}=1.56[1.14,2.13]$; although the BF computed was 0.194 , indicating support for the null hypothesis.

\section{Exploratory analyses}

A binomial logistic regression was performed to identify predictors of limit setting in response to messages. Four variables included in the model were significant predictors of limit setting (see Figure 3). Previous use of the deposit limit feature was the strongest predictor and being a customer of Operator 2 (compared to Operator 1) was associated with a decreased likelihood. The predictive ability $(\beta)$ for the two remaining significant predictors (i.e., betting intensity and age) was minimal. The overall model explained $61.8 \%$ of the variance in limit setting (Nagelkerke $R^{2}$ ) and its ability to predict this outcome was statistically significant compared to a null model $\left(n=26276, p_{<} .001\right)$.

Using robust ANCOVA analyses, we compared the change in three expenditure variables from pre- to post-limit (or pre/post-message for non-limit-setters) for limit setters and nonlimit-setters (the distribution of these variables for groups at both time points is visualised in Figure 4). The change in average daily wager amount was found to be significantly greater for limit setters at design points (i.e., baseline average daily wager values) of $\$ 6.02\left(\mathrm{n}^{l s}=83, \mathrm{n}^{n l}=81, M^{\operatorname{diff}(t r)}\right.$ $\left.=24.7,95 \% \mathrm{CI}[8.0,41.3], F=3.93, p_{<} .001\right)$ and $\$ 45.96$ $\left(\mathrm{n}^{l s}=89, \mathrm{n}^{n l}=100, M^{\operatorname{diff}(t r)}=28.9,[8.7,49.0], F=3.79\right.$, $\left.p_{<} .001\right)$, but not at $\left.\$ 94.33 p_{=} 0.004\right), \$ 220.97\left(p_{=} 0.908\right)$ or $\$ 384.95\left(p_{=} 0.144\right)^{6}$. The change in the standard deviation $(S D)$ of average daily wager was found to be significantly greater for limit setters at design points of $\$ 3.28\left(\mathrm{n}^{l s}=82\right.$, $\left.\mathrm{n}^{n l}=84, M^{\text {diff }(t r)}=29.4,[11.0,47.7], F=4.25, p_{<} .001\right)$, $\$ 39.98\left(\mathrm{n}^{l s}=88, \mathrm{n}^{n l}=100, M^{\operatorname{diff}(t r)}=30.1,[10.8,49.4], F\right.$ $\left.=4.12, p_{<} .001\right)$, and $\$ 78.43\left(\mathrm{n}^{l s}=92, \mathrm{n}^{n l}=107, M^{\text {diff }(t r)}=\right.$ $\left.30.0,[9.5,50.5], F=3.85, p_{<} .001\right)$, but not at $\$ 163.90$ ( $p_{=}$ $0.561)$ or $\$ 325.00\left(p_{=} 0.968\right)$. The change in net loss (i.e., total sum wagered minus winnings) was found to be significantly greater for limit setters at design points of $\$-431.88$ $\left(\mathrm{n}^{n l}=31, \mathrm{n}^{l s}=66, M^{\operatorname{diff}(t r)}=-335.1,[-512.0,-158.3], F=\right.$

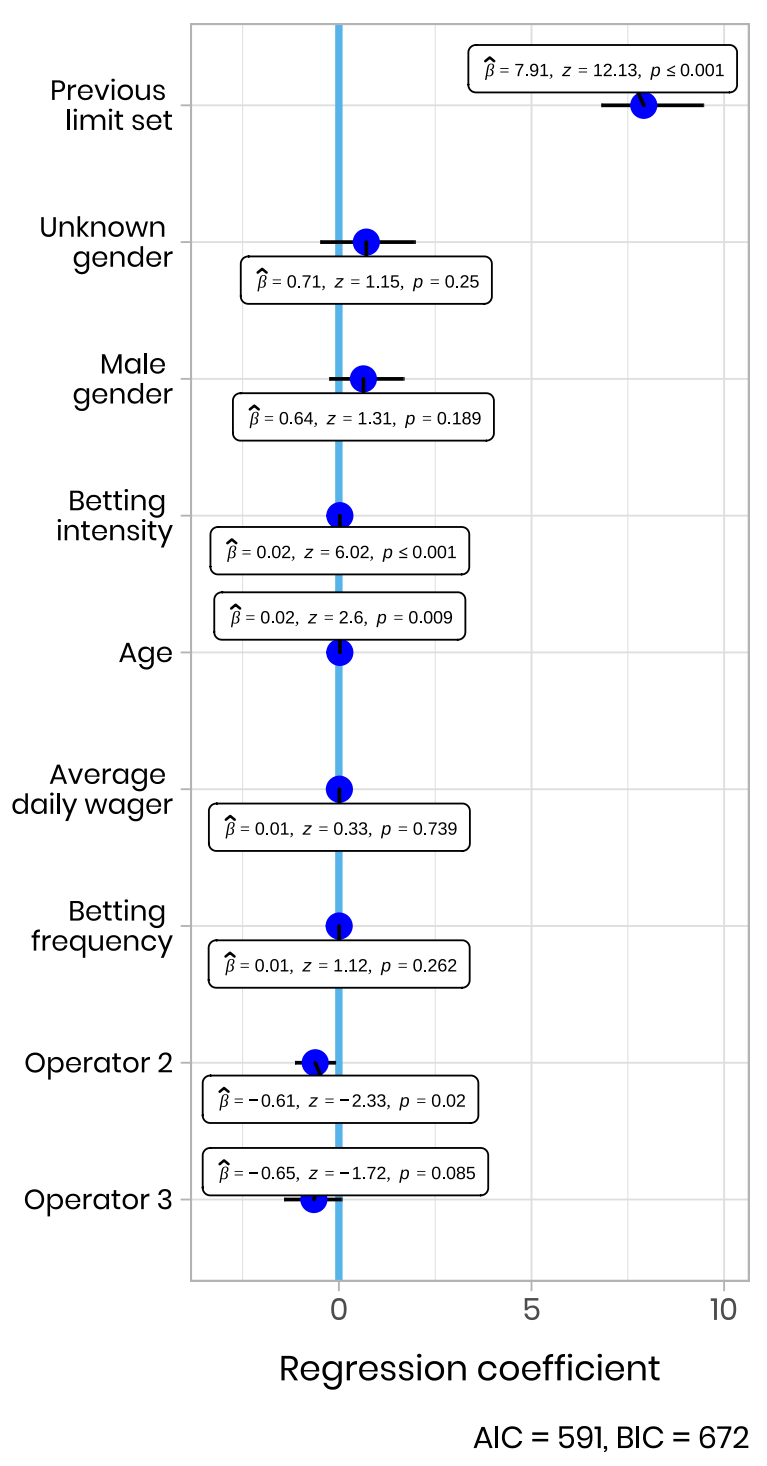

Figure 3. Predictors of limit setting in response to messages: Binomial logistic regression coefficients with $95 \%$ CIs. Figure legend: This figure displays the regression coefficients $(\beta)$ and their 95\% CIs for each predictor variable in the model, as well the associated $z$ (i.e., $\beta$ divided by its standard error) and $p$-values (calculated using Wald's test). The larger the regression coefficient, the more that a variable was predictive of limit setting. For the genderspecific predictor variables, females were the reference group. For the operator-specific predictor variables, Operator 1 was the reference group. AIC = Akaike information criterion; $\mathrm{BIC}=$ Bayesian information criterion.

\footnotetext{
${ }^{6}$ Robust ANCOVA outcome components and interpretation: $\mathrm{n}^{l s}$ $=$ limit setter sample size; $\mathrm{n}^{n l}=$ non-limit setter sample size; $M^{d i f f(t r)}=$ trimmed mean difference between groups change score; while we have denoted the test statistic using $F$, this value is not equivalent to the standard $F$ statistic; to save space, we have not reported the full outcomes of non-significant comparisons but they are shared on our OSF page.
} 
5.03, $\left.p_{<} .001\right), \$ 66.15\left(\mathrm{n}^{n l}=82, \mathrm{n}^{l s}=75, M^{\operatorname{diff}(t r)}=-256.60\right.$, $\left.[-434.5,-78.7], F=3.83, p_{<} .001\right)$, and $\$ 219.75\left(\mathrm{n}^{n l}=87, \mathrm{n}^{l s}\right.$ $\left.=75, M^{\operatorname{diff}(t r)}=-266.50,[-444.4,-88.6], F=3.97, p_{<} .001\right)$,

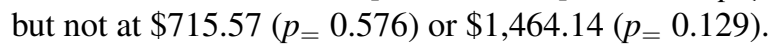

We also compared the change in two gambling behavioural variables for limit setters and non-limit-setters (the distribution of behavioural variables for groups at both time points is visualised in Figure 5). The change in betting frequency (i.e., the total number of days in the period in which at least one wager was placed) was not significantly different between groups at any design point: $5\left(p_{=} 0.018\right), 11\left(p_{=} 0.015\right), 19$ $\left(p_{=} 0.008\right), 31\left(p_{=} 0.219\right)$, or $67\left(p_{=} 0.122\right)$ days. The change in betting intensity (i.e., the total number of bets placed divided by betting frequency) was found to be significantly greater for limit setters at $1\left(\mathrm{n}^{n l}=82, \mathrm{n}^{l s}=61, M^{\text {diff }(t r)}=\right.$ -1.80, [-3.0, -0.6], $\left.F=3.93, p_{<} .001\right), 2.36\left(\mathrm{n}^{n l}=94, \mathrm{n}^{l s}=\right.$ $\left.70, M^{\text {diff }(t r)}=-2.2,[-3.6,-0.8], F=4.16, p_{<} .001\right)$, and 4.10 $\left(\mathrm{n}^{n l}=105, \mathrm{n}^{l s}=77, M^{\text {diff }(t r)}=-2.3,[-3.8,-0.8], F=4.14\right.$, $\left.p_{<} .001\right)$, but not at $7.79\left(p_{=} 0.003\right)$ or $16.92\left(p_{=} 0.959\right)$.

\section{Discussion}

One aim of this naturalistic RCT was to explore the value of different customer messages for increasing the uptake of deposit limits. Consistent with $\mathrm{H}^{1}$, customers who were sent messages were significantly more likely to set a deposit limit in the ensuing five days compared to controls. Message content had little effect on uptake rates, inconsistent with our prediction in $\mathrm{H}^{2}$. Messages delivered via in-account notification were more effective at increasing uptake than email messages, supporting $\mathrm{H}^{3}$; although the Bayesian test of this relationship indicated there was little association between delivery method and limit setting, suggesting further research comparing the impact of different message delivery methods is required.

Considering all messaging conditions together, customer messages resulted in a small but notable increase $(0.71 \%)$ in the use of the deposit limit feature, rising to $1.13 \%$ when considering email opening rates. This is less than rates reported by Behavioural Insights Team (14), who observed uptake rates for deposit limits of between 3.3 and $4.4 \%$ in response to their messages. There are two possible reasons for this discrepancy. First, they sent their messages in response to the operators' risk-identification system alerting them to a customer displaying risk behaviours (e.g., use of multiple payment methods), suggesting these individuals may have perceived assistive tools as more relevant at the time of messaging. Second, all online gambling sites in Australia are required, as of May 2020 (five months before the start of the trial), to make their customers set a deposit limit or explicitly opt-out of setting one. Thus, individuals involved in this trial will have already opted-out of setting a limit or-as was
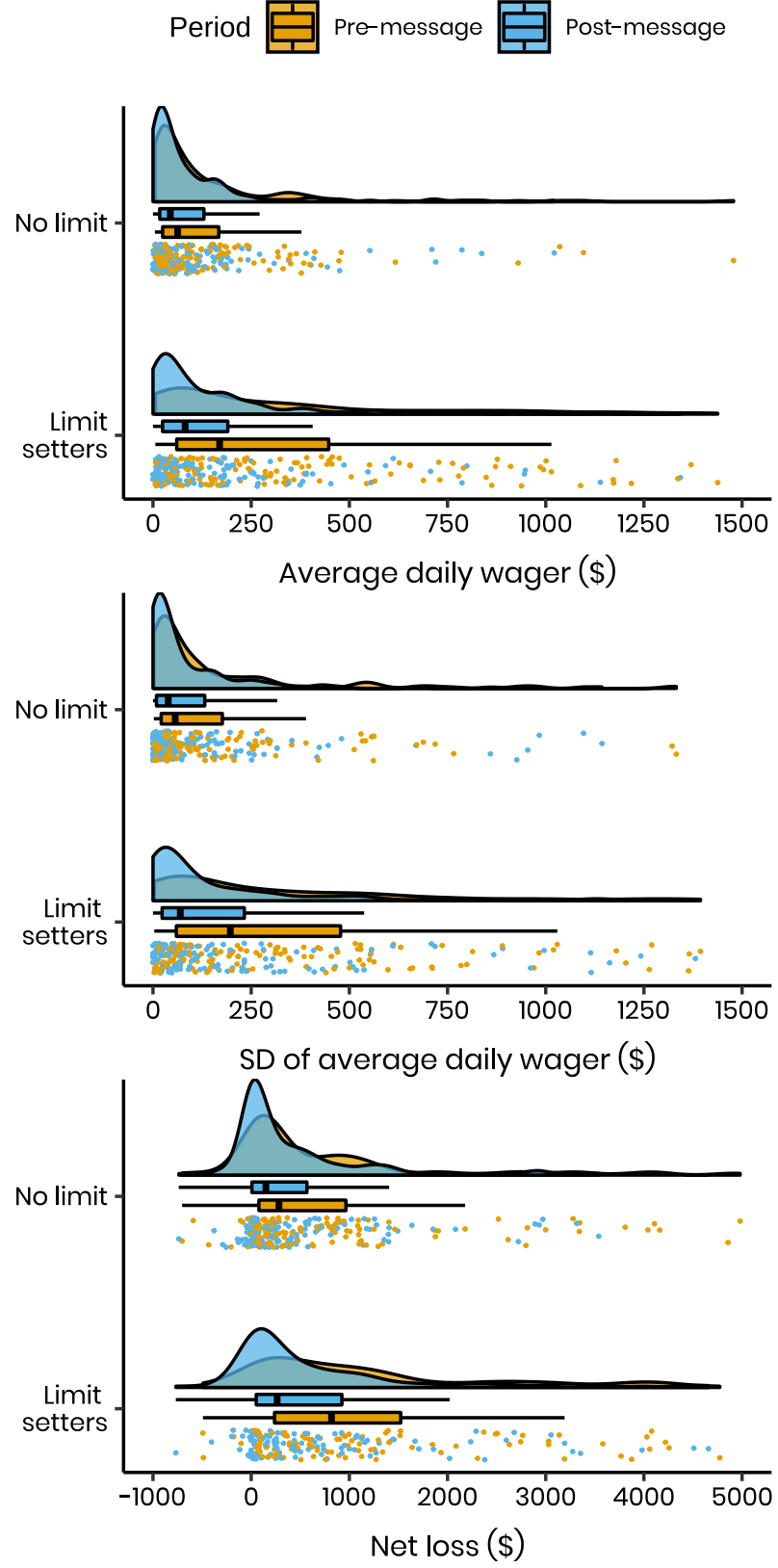

Figure 4. Effects of deposit limits on wagering expenditure (90 days before $\&$ after messages). Figure legend: This figure displays the distribution of customers' average daily wager amount (a), the variability (SD) in average daily wager (b), and net loss (c) for the 90 days before and after receiving the first message for nonlimit-setters and 90 days before and after setting their limit for the those who set a limit. The scale of Figure $4 \mathrm{a}$ and $4 \mathrm{~b}$ were capped at $\$ 1,500$ and Figure $4 \mathrm{c}$ was capped at $\$-1,000$ and $\$ 5,000$ to enable easier visualisation of outcomes. The non-truncated versions of the plots are shared on OSF. 

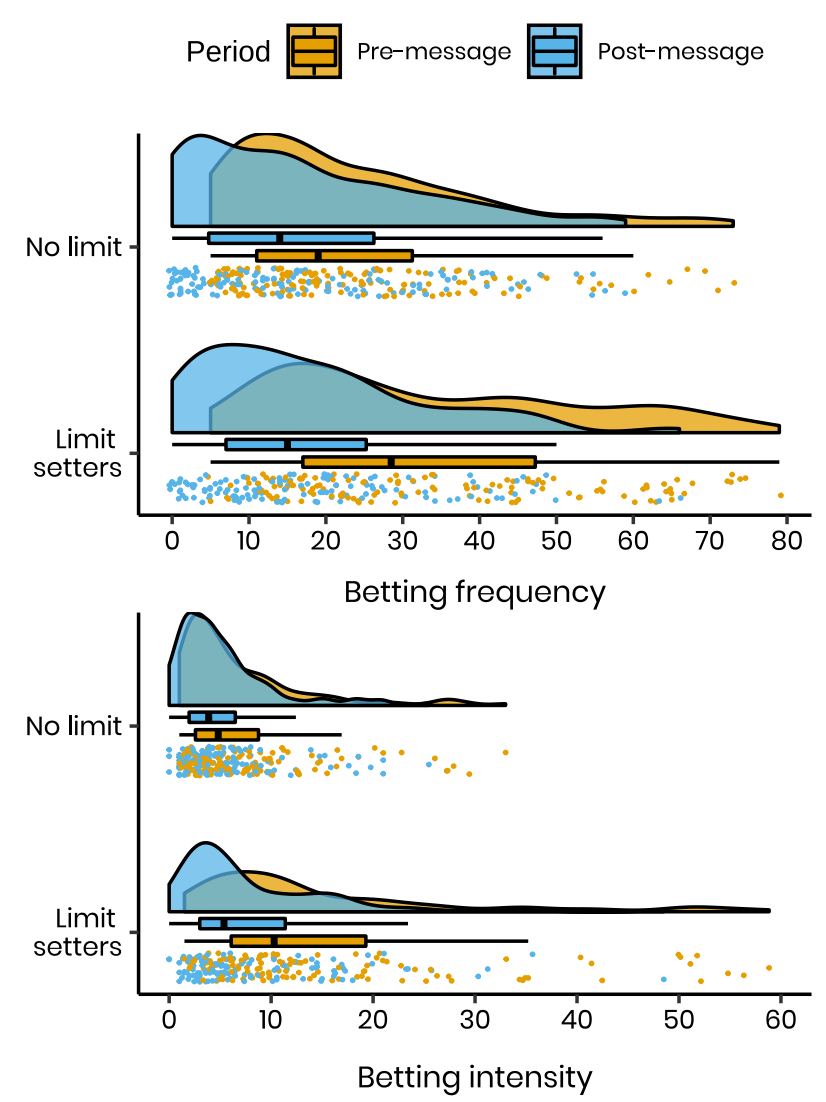

Figure 5. Figure 5: Effects of deposit limits on wagering behaviour (90 days before $\&$ after). Figure legend: This figure displays the distribution of customers' betting frequency (a) and intensity (b) for the 90 days before and after receiving the first message for non-limit-setters and 90 days before and after setting their limit for the those who set a limit. The scale of Figure 5b was capped at 60 to enable easier visualisation of outcomes. The non-truncated version of the plot is shared on OSF.

frequently the case—removed a previously set limit. Indeed, having previously set a limit was the strongest predictor of setting one in response to messages, suggesting customer messages may be a useful strategy for encouraging re-uptake of CPTs.

In relation to the second aim, the study provides important results to support the value of deposit limits in shifting customers to sustainable gambling behaviours. Monitoring gambling expenditure and involvement revealed that limit setters, compared to non-limit-setters, showed a significantly greater reduction in average daily wager, variation in average daily wager, net loss, and betting intensity. Specifically, individuals with low-mid levels of these variables (e.g., an average daily wager of between $-\$ 6 \& \sim \$ 94$ ) in the 90-days preceding messages showed a significantly greater reduction compared to non-limit-setters with similar baseline values, whereas the change in values displayed by limit setters with higher baseline values (e.g., average wager of $\sim 220 \& \sim \$ 384$ ) was not significantly different from nonlimit-setters with similar baseline values. However, it is unknown whether deposit limit users migrated to other sites and therefore did not reduce their overall gambling expenditure and involvement. Nonetheless, these findings are consistent with extant studies which have observed reductions in betting involvement and expenditure following limit setting (57), but diverge from Ivanova et al. (8) who found no effect of limit setting on net loss. This discrepancy may be because these authors compared net loss in the 90-days after registration between limit setters and non-limit-setters, as opposed to the change in net loss observed before and after limit setting, as was done here.

This is the first study to have studied both the effects of consumer messages and the effects of limit setting on online gambling sites in Australia. The naturalistic RCT design used and the involvement of multiple operators adds credence to the external validity of the findings observed. However, as the study involved Australian online wagererswhose gambling activity is restricted to sports and race wagering - customers in other jurisdictions and/or using alternative forms of online gambling may respond differently to the messages used. Further, we targeted cohorts of individuals who engaged in regular gambling, but without consideration of behavioural risk indicators like the Behavioural Insights Team's 2018 study (14). Instead, we aimed to encourage deposit limit use as a harm reduction strategy for all regular online wagering customers as this may be a method of preventing the development of problems. Allaying any concerns that the efforts to encourage CPT use in this study or by gambling sites in the future may lead to high rates of irritation or site abandoning, research has found customers are not inconvenienced by the promotion of CPTs, including those at different risk levels for gambling problems (3). Future research in live gambling environments is required to determine the effectiveness of targeted messages sent in response to certain risk behaviours (14), as well as messages tailored to individuals and based on cohort preferences, both of which have shown preliminary value as customisation strategies $(23,24)$.

Overall, the findings from this study suggest customer messaging may be an inexpensive and easily implemented strategy online gambling sites can use to increase the use of deposit limits and other CPTs. Our findings add to existing evidence which implies the way in which messages are delivered and presented to customers may be more important than the content (14), though further comparisons of different message content are required. Given the reductions in gambling expenditure and involvement observed among limit setters in this study, the provision and promotion of deposit limits should be encouraged on online sites as a possible harm 
reducing strategy.

\section{Funding sources \& conflicts of interest}

The authors declare no conflicts of interest. This study was partially funded by Responsible Wagering Australia (RWA), a body representing the Australian online wagering industry. RWA member operators provided data based on specific requirements of the research team. SG's contribution was also supported by a Discovery Early Career Research Award (DECRA DE1060100459) provided by the Australian Research Council (ARC). RWA and the ARC were not involved in the design of the study, the analysis or interpretation of findings, writing or approving the final manuscript for publication.

\section{References}

1. Gainsbury SM, Angus DJ, Procter L, Blaszczynski A. Use of Consumer Protection Tools on Internet Gambling Sites: Customer Perceptions, Motivators, and Barriers to Use. Journal of Gambling Studies. 2019 May;36:259-76.

2. Griffiths MD, Wood RTA, Parke J. Social Responsibility Tools in Online Gambling: A Survey of Attitudes and Behavior among Internet Gamblers. CyberPsychology \& Behavior. 2009 Jul;12(4):413-21.

3. Ivanova E, Rafi J, Lindner P, Carlbring P. Experiences of responsible gambling tools among non-problem gamblers: A survey of active customers of an online gambling platform. Addictive Behaviors Reports. 2019 Jun;9:100161.

4. Auer M, Reiestad SH, Griffiths MD. Global limit setting as a responsible gambling tool: What do players think? International Journal of Mental Health and Addiction. 2018;1-13.

5. Nelson SE, LaPlante DA, Peller AJ, Schumann A, LaBrie RA, Shaffer HJ. Real limits in the virtual world: Self-Limiting behavior of Internet gamblers. Journal of Gambling Studies. 2008 Dec;24(4):463-77.

6. Auer M, Griffiths MD. Voluntary limit setting and player choice in most intense online gamblers: An empirical study of gambling behaviour. Journal of Gambling Studies. 2013 Dec;29(4):647-60.

7. Auer M, Hopfgartner N, Griffiths MD. The Effects of Voluntary Deposit Limit-Setting on Long-Term Online Gambling Expenditure. Cyberpsychology, Behavior, and Social Networking. 2020 Feb;23(2):113-8.

8. Ivanova E, Magnusson K, Carlbring P. Deposit Limit Prompt in Online Gambling for Reducing Gam- bling Intensity: A Randomized Controlled Trial. Frontiers in Psychology. 2019;10.

9. UKGC. Gambling participation in 2018: Behaviour, awareness and attitudes. UK: UK Gambling Commission; 2019.

10. Procter L, Gainsbury SM, Angus DJ, Blaszczynski A. Understanding use of consumer protection tools among Internet gambling customers: Utility of the Theory of Planned Behavior and Theory of Reasoned Action. Addictive Behaviors. 2019 Jul;106050.

11. Ajzen I. From Intentions to Actions: A Theory of Planned Behavior. In: Kuhl J, Beckmann J, editors. Action Control: From Cognition to Behavior. Berlin, Heidelberg: Springer Berlin Heidelberg; 1985. pp. 11-39. (SSSP Springer Series in Social Psychology).

12. Fishbein M, Ajzen I. Belief, attitude, intention and behavior: An introduction to theory and research. Reading, MA: Addison-Wesley; 1975.

13. Bagot KL, Cheetham A, Lubman DI, Rodda SN. Predictors of Strategy Engagement for the Prevention and Reduction of Gambling Harm: A Prospective Application of the Theory of Planned Behaviour. International Journal of Mental Health and Addiction. 2020 Apr;

14. BIT. Can behavioural insights be used to reduce risky play in online environments? [online] https://about.gambleaware.org/media/1869/gambleawarephase-iii-report_updated-v1.pdf; 2018.

15. Scheel AM, Tiokhin L, Isager PM, Lakens D. Why Hypothesis Testers Should Spend Less Time Testing Hypotheses. Perspectives on Psychological Science. 2020 ;in press.

16. Gaglio B, Phillips SM, Heurtin-Roberts S, Sanchez MA, Glasgow RE. How pragmatic is it? Lessons learned using PRECIS and RE-AIM for determining pragmatic characteristics of research. Implementation Science. 2014 Aug;9:1-11.

17. Haff N, Choudhry NK. The Promise and Pitfalls of Pragmatic Clinical Trials for Improving Health Care Quality. JAMA Network Open. 2018 Oct;1(6):e183376-6.

18. Simon S. How to randomise. BMJ (Clinical Research Ed). 1999;319(703).

19. R Core Team. R: A language and environment for statistical computing. Vienna, Austria: R Foundation for Statistical Computing; 2020. 
20. Zhang S, Paul J, Nantha-Aree M, Buckley N, Shahzad $\mathrm{U}$, Cheng J, et al. Empirical comparison of four baseline covariate adjustment methods in analysis of continuous outcomes in randomized controlled trials. Clinical epidemiology. 2014 Jul;6:227-35.

21. Mair P, Wilcox R. Robust statistical methods in R using the WRS2 package. Behavior Research Methods. 2020 Apr;52(2):464-88.

22. Wilcox R. Chapter 11 - More Regression Methods. In: Wilcox R, editor. Introduction to Robust Estimation and Hypothesis Testing (Third Edition). Boston: Academic Press; 2012. pp. 533-629.

23. Auer M, Griffiths MD. The use of personalized messages on wagering behavior of Swedish online gamblers: An empirical study. Computers in Human Behavior. 2020 Sep;110:106402.

24. Gainsbury SM, Abarbanel BLL, Philander KS, Butler JV. Strategies to customize responsible gambling messages: A review and focus group study. BMC Public Health. 2018 Dec;18(1). 